\title{
Les kinoïdes
}

\section{Une nouvelle génération de vaccins thérapeutiques}

Armand Bensussan, Bernard Bizzini, Philippe Pouletty, Robert C. Gallo, Daniel Zagury

$M / S$ inaugure ici une série d'articles consacrés aux stratégies scientifiques de sociétés de biotechnologie. Nous tenons à remercier les entreprises se prêtant à cet exercice difficile et tout particulièrement leurs directeurs scientifiques. S'agissant d'entreprises commerciales, et de paris sur l'avenir, il sera aisé à nos lecteurs de comprendre que ces articles ne peuvent être écrits avec le recul critique habituellement de mise pour tout auteur scientifique. Ils seront lus dans ce contexte. M/S assume le concept de conflit d'intérêt en ce qu'il peut conduire à une présentation excessivement affirmative, positive et univoque des éléments rationnels sur lesquels s'appuie la démarche de l'entreprise. Comme pour tout article que nous publions, nos colonnes sont ouvertes aux lecteurs souhaitant publier une « Répartie » scientifiquement argumentée aux propos tenus ci-après.

Hervé Chneiweiss Rédacteur en chef (France)

> Depuis maintenant plus d'un siècle, les vaccins font partie de l'arsenal thérapeutique servant à combattre et maîtriser un grand nombre d'infections. À la faveur des progrès biotechnologiques et d'une meilleure connaissance de la physiopathologie associée à certaines affections chroniques, l'innocuité, l'immunogénicité et l'efficacité des préparations vaccinales ont été améliorées (épitopes antigéniques mieux ciblés, adjuvants non toxiques...). Malgré ces efforts, l'approche vaccinale thérapeutique conventionnelle reste inefficace dans certains cas comme le Sida ou les cancers. Pour expliquer ces échecs thérapeutiques, nous avons postulé que l'échappement immunitaire des cellules malades, infectées par un virus ou tumorales, pouvait être lié à des dysfonctionnements cytokiniques pouvant contribuer à une paralysie immunitaire. <

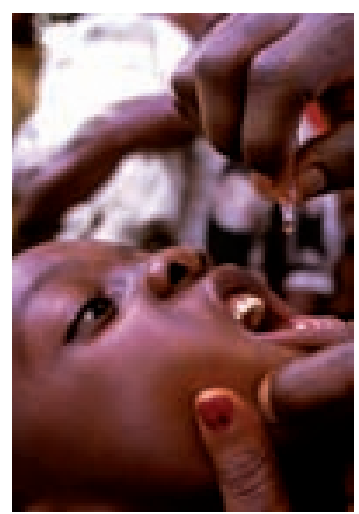

A. Bensussan : Institut National de la Santé et de la Recherche Médicale, Inserm U841, Créteil, France; Institut Mondor de Médecine Moléculaire, Hôpital Henri Mondor, 51, avenue du Maréchal de Lattre de Tassigny, 94010 Créteil, France. armand. bensussan@creteil.inserm.fr B. Bizzini, P. Pouletty, D. Zagury: Neovacs SA, 3-5, impasse Reille, 75014 Paris, France.

Les vaccins thérapeutiques conventionnels

\section{dzagury@neovacs.com}

R.C. Gallo : Institute of Human Virology, University of Maryland, 725 West Lombard Street,

Les vaccins sont couramment utilisés avec Suite S307, Baltimore, MD 21201, États-Unis.

succès depuis plus d'un gallo@umbi.umd.edu siècle dans le traitement ou la prévention de maladies infectieuses. Leur mécanisme d'action est physiologique et consiste à initier et développer une réaction immunitaire contre toute substance ou structure porteuse d'un antigène (virus, bactérie, cellule tumorale), considérée comme non-soi par l'organisme de manière à promouvoir un état immun, une mémoire immunitaire, vis-à-vis de l'antigène étranger. La réaction immunitaire induite par ces vaccins conventionnels comprend une production d'anticorps neutralisant l'agresseur antigénique circulant et une réponse cellulaire effectrice, avec la différenciation de cellules cytotoxiques capables de détruire les cellules malades porteuses de l'antigène étranger. L'extension à des fins thérapeutiques de l'approche vaccinale utilisée ces dernières années dans le Sida (antigènes du VIH) [1-3] et dans certains cancers (antigènes tumoraux associés TAA, ou spécifiques de tumeurs TSA, des cellules cancéreuses) n'est pas apparue, à ce jour, efficace [4]. Aussi le vaccin quadrivalent dirigé contre le HPV (human papillomavirus), validé cliniquement et efficace, est aujourd'hui prescrit à titre préventif chez les personnes non infectées par l'agent viral causal de la maladie 
[38], mais n'est pas indiqué en l'état pour usage thérapeutique chez les patientes qui souffrent de métaplasies ou de dysplasies du col utérin induites par le virus (Figure 1) [5].
Les dysfonctionnements cytokiniques :

éléments pathogènes

de maladies chroniques sévères
A

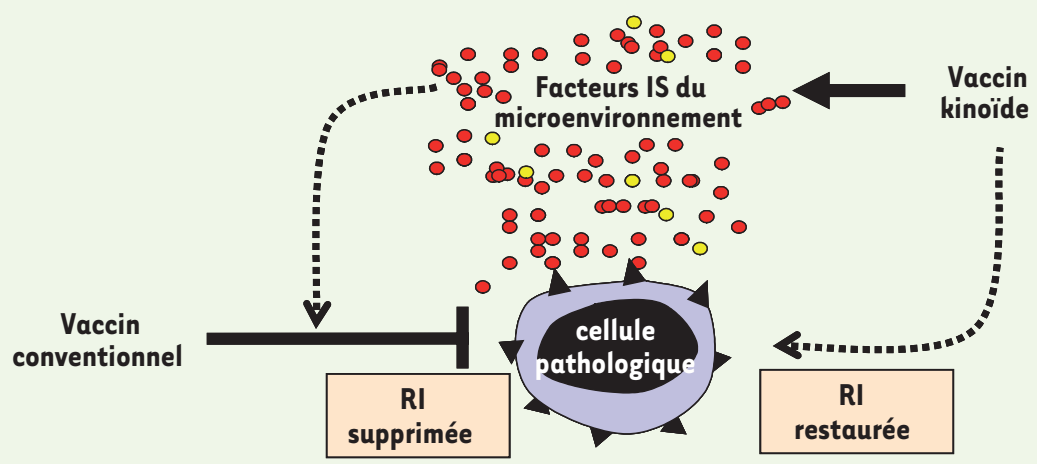

B
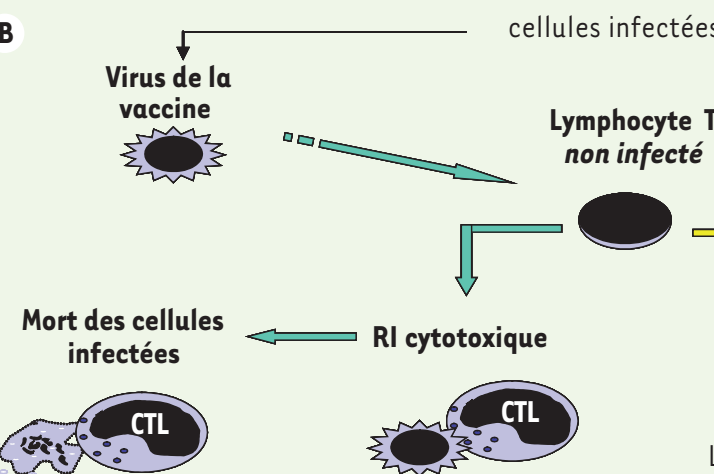

RI cytotoxique

C

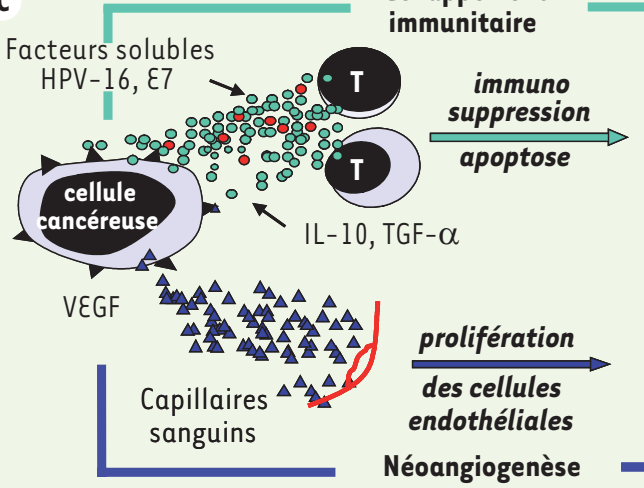

\begin{abstract}
(1)
\end{abstract}
Lymphocyte T
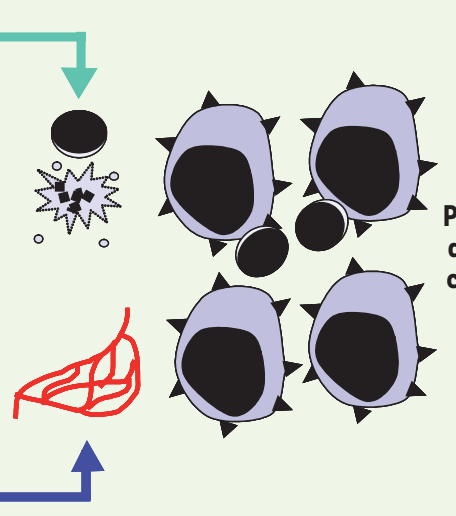

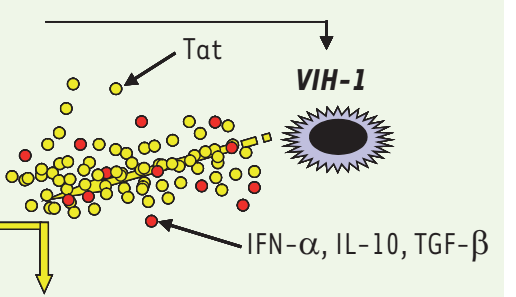

RI suppressive $\Longrightarrow$ Réplication et propagation du VIH

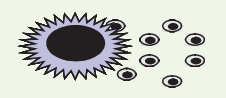

Prolifération des cellules cancéreuses

Figure 1. A. Cibles vaccinales visées par Neovacs. Le vaccin conventionnel cible uniquement les antigènes anormaux présents à la surface des cellules pathologiques et induit préférentiellement une réponse cellulaire tueuse contre ces cellules. Cette réponse immunitaire (RI) peut être supprimée par des facteurs du microenvironnement. Les vaccins kinoïdes en induisant des anticorps visent à neutraliser les cytokines ectopiques immunosuppressives (IS) et/ou angiogéniques, délétères du microenvironnement stromal des tissus pathologiques et peuvent ainsi permettre au vaccin conventionnel ou aux défenses naturelles d'exercer leur effet anti-tumoral. B et $C$. $\varepsilon f f e t s$ pathogènes induits par les protéines pathogènes du microenvironnement des tissus pathologiques. B. Combat déséquilibré entre le virus VIH-1 et le système immunitaire de l'hôte : les cellules infectées par le VIH relâchent la protéine Tat, laquelle stimule les cellules immunitaires (CPA et T) qui produisent des cytokines à effet immunosuppressif (IFN- $\alpha$, IL-10, TGF- $\beta$ ) inhibant la réponse immunitaire antivirale. C. Induction d'immunosuppression et de néoangiogenèse intratumorales. Les cellules cancéreuses ou stromales de tumeurs relâchent des facteurs immunosuppresseurs : protéines virales ( $(7)$, ou cellulaires (TGF- $\beta$, IL-10) et angiogéniques (VEGF).
Les cytokines sont des signaux intercellulaires qui jouent un rôle majeur dans la physiopathologie des réactions immunitaires comme cela est décrit dans l'Encadré. Leur rôle délétère, au cours d'affections virales ou cancéreuses, est étayé par un ensemble d'observations expérimentales et cliniques. Contrairement à la majorité des virus, comme ceux de la vaccine ou de l'influenza qui activent des réponses immunitaires cytotoxiques contrôlant l'infection, le VIH entraîne une production anormale d'IFN- $\alpha$ (interféron- $\alpha$ ) [6], d'IL-10 (interleukine-10) [7] et de TGF$\beta$ (transforming growth factor- $\beta$ ) [8], associée à une immunosuppression des cellules $T$ conduisant à la maladie Sida. La production ectopique de I'IFN- $\alpha$, non contrôlée homéostatiquement, peut être due à l'activation des cellules productrices d'IFN- $\alpha$ ( $p D C$, cellules dendritiques plasmacytoïdes, et autres cellules présentatrices d'antigènes [CPA]) par la protéine virale Tat, excrétée par les cellules infectées (Figure 1B) [9]. Dans le cancer du col utérin, la protéine $\varepsilon 7$ de l'HPV-16, excrétée par les cellules cancéreuses, active non seulement une production anormale d'IFN- $\alpha$ à effet immunosuppresseur par les CPA 
[10], mais aussi la prolifération des cellules endothéliales qui sous tend l'angiogenèse tumorale [11] (Figure 1C). Dans d'autres types de tumeurs, les cellules malignes sécrètent directement des cytokines à action immunosuppressive, susceptibles de paralyser localement les réactions immunitaires, ou ayant d'autres effets pathogènes, tels que le VEGF (vascular endothelial growth factor) excrété par de nombreuses cellules cancéreuses, qui induit la néoangiogenèse tumorale [12]. Le dysfonctionnement cytokinique au sein des tissus pathologiques s'observe également dans d'autres pathologies; c'est le cas de la polyarthrite rhumatoïde caractérisée cliniquement par l'inflammation des articulations et biologiquement par une surproduction de cytokines pro-inflammatoires (TNF $\alpha$, IL-1, IFN- $\gamma$ ) [13].

\section{La vaccination kinoïde, nouvelle arme thérapeutique contre les dysfonctionnements cytokiniques}

Introduits dès 1991 par le groupe de recherche d'immuno-physiologie cellulaire de l'Université Pierre et Marie Curie (UPMC, Paris VI, France), les vaccins kinoïdes visent à produire des anticorps de haute affinité dirigés contre les cytokines ectopiques anormalement présentes dans le stroma des tissus pathologiques pour en inhiber les effets pathogènes et permettre aux cellules immunitaires, activées spontanément ou après vaccination conventionnelle, d'exercer leur fonction effectrice tueuse vis-à-vis des cellules pathologiques (Figure 1). De tels vaccins sont constitués de dérivés de ces cytokines, biologiquement non toxiques, mais immunogènes [14]. La société Neovacs (Encadré 2) a engagé depuis 1993 un programme de développement industriel de ces vaccins.

\section{(1)}

Les cytokines: signaux de communication intercellulaire du système immunitaire

Les cytokines sont des signaux assurant l'homéostasie des tissus. Elles sont généralement produites de manière inductible par des cellules effectrices. Ces cellules agissent localement de manière paracrine ou autocrine dans le cadre de champs cytokiniques sur des cellules cibles porteuses de récepteurs spécifiques en activant les voies de signalisation intracellulaires de ces dernières. Les cellules du système immunitaire communiquent entre elles via des cytokines. Ainsi, au niveau d'un champ cytokinique, comportant une synapse immunitaire CPA-lymphocyte T, les cytokines produites par la cellule effectrice CPA exerceront sur la cellule T cible-répondeuse une action variable, dépendante du contexte intracellulaire de cette dernière. De fait, par les voies de signalisation qu'elles activent sur la cellule cible immunitaire, les cytokines contribuent à en réguler les différentes fonctions: activation (IL-2, IL-6, TNF- $\alpha$, IFN- $\alpha$ ), prolifération (IL-2, IL-4, IFN- $\gamma$ ), différenciation (IFN- $\gamma$, IL-6, IL-10), survie cellulaire (IL-2, IFN- $\gamma$, IFN$\alpha$ ), mort programmée (TRAIL, FasL, TNF- $\alpha$, IFN- $\alpha$ ), et chimiotactisme (MIP1- $\alpha / C C L 3$, RANTES/CCL5, SDF-1/CCL12). Les dysfonctionnements physiopathologiques cytokiniques contribuent ainsi à la pathogenèse de maladies chroniques graves [2].

\section{(2)}

\section{La société Neovacs}

La société Neovacs créée en 1993 comme une spin-off de l'Université Pierre et Marie Curie, est spécialisée dans le développement de vaccins thérapeutiques et détient un large portefeuille de brevets industriels relatif à ces vaccins. Cette biotech est en particulier reconnue comme pionnière dans le développement des vaccins Kinoïdes. Ces derniers visent à inhiber les effets pathogènes de cytokines anormalement présentes dans le microenvironnement stromal des tissus pathologiques de maladies chroniques, virales, cancéreuses ou auto-immunes. Une fois validée, cette seconde génération d'immunothérapie spécifique anticytokine, peu contraignante, pourra représenter une alternative à l'administration passive d'anticorps monoclonaux utilisés aujourd'hui en clinique dans le traitement des polyarthrites rhumatoïdes, de la maladie de Crohn et de certains cancers.

\section{Les vaccins kinoïdes}

Le kinoïde, qui représente le principe actif du vaccin, est un immunogène capable d'induire des autoanticorps de haute affinité contre une cytokine donnée [15]. Dans sa composition usuelle, il s'agit d'un hétérocomplexe obtenu par liaison chimique de la cytokine à une protéine porteuse étrangère telle que le KLH (Keyhole Limpet Hemocyanin) [16]. La protéine porteuse KLH a un rôle double, celui de fournir les déterminants antigéniques (épitopes T) nécessaires à l'activation des cellules $T$ auxiliaires et celui de présenter aux cellules B productrices d'anticorps une densité élevée d'antigènes cytokiniques dans leur conformation native. Notons que le KLH est actuellement utilisé à des doses élevées comme immunostimulant dans des essais cliniques en cancérologie (cancer de la vessie) [17].

D'autres agents de couplage, comme le carbodiimide, ou d'autres protéines porteuses, comme l'ovalbumine, ont été utilisés pour produire avec succès des kinoïdes dirigés contre I'IL-9 et I'IL-17 [18, 19]. D'autres vaccins kinoïdes ont été préparés à partir d'immunogènes constitués d'un pool de protéines mutées analogues de la cytokine IL-6 native [20], ou encore à partir de vecteurs auxiliaires lipidiques tels que les VLP (virus like particles) anti-TNF (tumor necrosis factor) [21]. Dans certaines situations expérimentales (chez l'animal après stimulation vaccinale en présence de LPS ou de $\mathrm{CpG}$ [ $[22,23]$ ou dans certains états pathologiques (Sida) [24], la cytokine seule après inactivation 
A

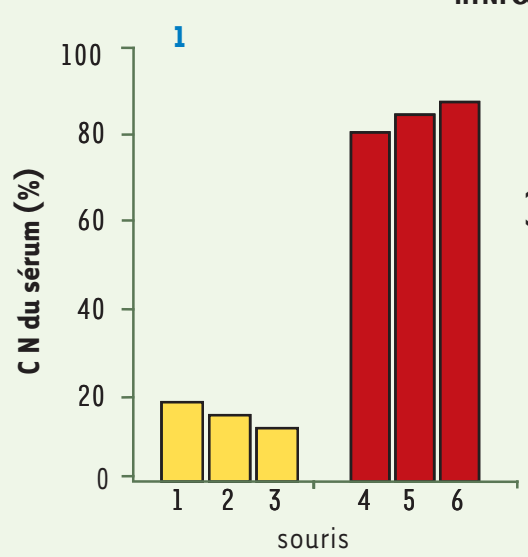

\section{hTNF $\propto$}

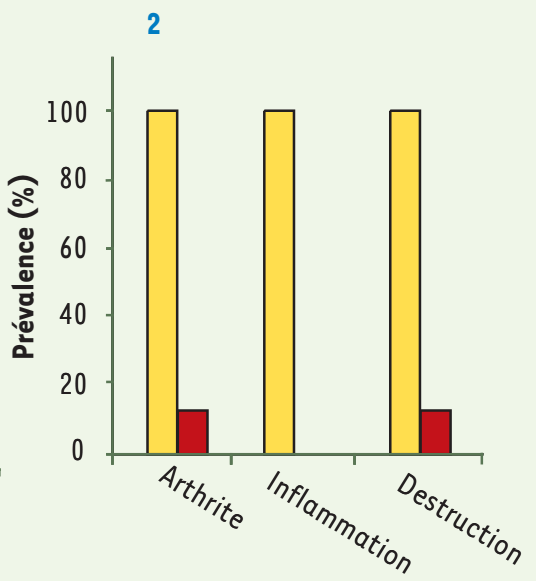

B

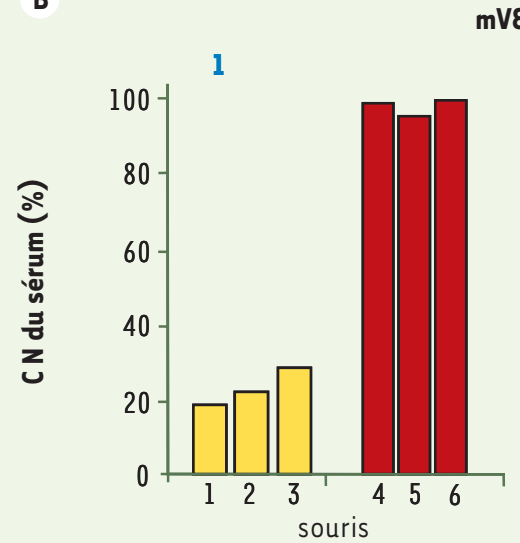

mVEGF

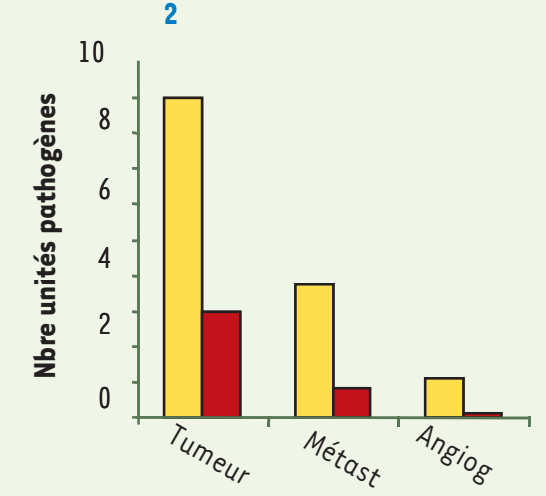

C

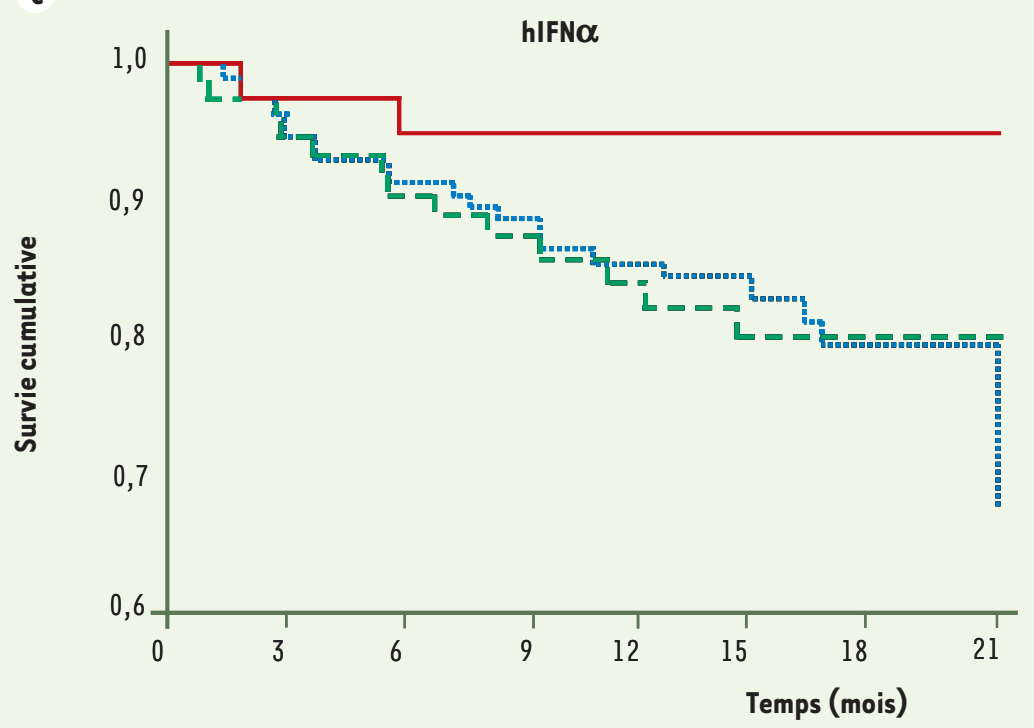

peut se substituer comme immunogène à l'hétérocomplexe kinoïde. La cytokine antigène dans ces situations particulières induit une réponse anticorps anticytokine, sans l'apport auxiliaire de la protéine carrier KLH (réponse B anticytokine T-indépendante).

Figure 2. Effets de la vaccination kinoïde. A1. Anticorps neutralisants induits par la vaccination kinoïde hTNF $\boldsymbol{\alpha}$. Inhibition de l'activité fonctionnelle du hTNF- $\alpha$, en présence de sérum de souris (dilution au $1 / 100^{\mathrm{e}}$ ) immunisées avec le kinoïde hTNF $\alpha$, évaluée par le test de cytotoxicité des cellules murines $L 929$ exprimée par le \% d'inhibition de la lyse cellulaire de la lignée L929 en présence de hTNF $\alpha$. CN : capacité neutralisante, barre rouge: groupe expérimental, barre jaune: groupe témoin. A2. Efficacité clinique associée au vaccin kinoïde hTNF $\alpha$. Effet de l'immunisation TNF- $\alpha$ kinoïde sur les scores cliniques et histologiques de l'arthrite chez les souris immunisées avec le kinoïde TNF- $\alpha$ (barre rouge) ou avec le KLH (barre jaune). La prévalence de l'inflammation/destruction évaluée par histologie est le pourcentage (\%) de souris avec un score d'inflammation/destruction $\geq 0,5$ (score allant de 0 [aspect normal] à 3 [inflammation sévère avec déformation de l'articulation]) (adapté de [14]). B1. Anticorps neutralisants induits par la vaccination kinoïde VEGF. Inhibition de l'activité fonctionnelle du VEGF, en présence de sérum de souris (dilution au $1 / 100^{e}$ ) immunisées avec le kinoïde mVદGF, évaluée par le test de prolifération de cellules HUVEC (human umbilical vein endothelial cells) en présence de VEGF. CN : capacité neutralisante, barre rouge: groupe expérimental, barre jaune: groupe témoin. B2. Efficacité clinique associée au vaccin kinoïde hVEGF. Effet de l'immunisation VEGF kinoïde sur les métastases pulmonaires de carcinome du côlon CT26 chez les souris immunisées avec le kinoïde mVEGF (barre rouge) et chez les souris immunisées avec le KLH (barre jaune). Fréquences d'unités pathogènes évaluées par: Tumeur: Score de tumeurs détectées macroscopiquement (absence $=0$ et présence $=1$ ); Métast : Moyenne du nombre de métastases détectées au

microscope ; Angiog: Score d'angiogenèse par métastase détectée au microscope (absence = 0 et présence =1) (adapté de [26]). C. $\varepsilon f f e t$ de l'immunisation IFN- $\alpha$ Kinoïde mesurée par la survenue de signes cliniques en relation avec le VIH et représentée sur la courbe Kaplan-Meier de survie cumulative dans le temps chez des patients infectés par le VIH-1. En abscisse, la durée du suivi clinique en mois et, en ordonnée, les événements en rapport avec l'infection VIH, trait plein rouge : sujets vaccinés répondeurs (présence d'anticorps anti-IFN $\alpha$ sérique), trait discontinu vert : sujets vaccinés non répondeurs, trait en pointillé bleu : sujets ayant reçu un placebo (adapté de [25]). 
À ce jour, la société Neovacs dispose de vaccins kinoïdes dirigés contre le TNF- $\alpha$ humain, le VEGF humain/murin, I'IL-4 humaine/murine, I'IFN$\alpha$ humain/murin. Ces vaccins ont été testés dans différents modèles animaux in vivo afin d'en évaluer l'immunogénicité, la tolérance et l'efficacité thérapeutique [14, 25-27].

\section{Les effets de la vaccination kinoïde}

L'immunisation avec le kinoïde émulsionné dans l'adjuvant huileux de Salk (ISA51-Seppic, Paris) induit des titres élevés d'anticorps neutralisants de haute affinité, dirigés contre la cytokine native correspondante. L'efficacité de ces anticorps anti-cytokine a été démontrée expérimentalement.

\section{TNF- $\boldsymbol{\alpha}$ Kinoïde}

Chez la souris transgénique exprimant le TNF- $\alpha$ humain (Tg1006-T de Taconic), qui développe une polyarthrite rhumatoïde, l'immunisation avec le kinoïde TNF- $\alpha$ humain induit des taux élevés d'auto-anticorps sériques de haute affinité (Figure $2 \mathrm{Al}$ ) et réduit de façon très importante les signes cliniques (oedèmes et déformation articulaire mesurée par un score arthritique) (Figure 2A2) et histologiques (inflammation et destruction osseuse) de la maladie.

\section{VعGF Kinoïde}

Le kinoïde VEGF murin induit chez la souris immunisée des titres élevés d'auto-anticorps neutralisants anti-VEGF (Figure 2B). Chez la souris Balb/c greffée avec des cellules cancéreuses syngéniques (lignée CT26), l'immunisation avec le kinoïde VEGF réduit significativement le nombre de métastases pulmonaires [26].

\section{IL-4 Kinoïde}

Chez la souris Balb/c sensibilisée à l'allergène rB et v la, l'immunisation avec le kinoïde IL-4 diminue sensiblement la réponse anticorps anti-rBet vla de classe Igદ. D’autre part, chez la souris sensibilisée à l'ovalbumine, l'immunisation avec le kinoïde IL-4 améliore la réaction inflammatoire pulmonaire induite par un challenge avec cet allergène [27].

\section{IFN- $\alpha$ Kinoïde}

Dans un modèle expérimental de lupus érythémateux disséminé, les souris NZB/W ayant reçu une injection d'adénovirus exprimant l'IFN- $\alpha$ murin développent une protéinurie suivie de la mort par glomérulonéphrite. L'immunisation de ces souris avec le kinoïde IFN- $\alpha$ murin prévient cette évolution ( $S$. Koutouzov, manuscrit en préparation). Chez l'homme, l'essai EURIS réalisé chez 242 sujets infectés par le VIH a montré un bénéfice clinique et biologique chez les sujets répondeurs présentant des titres élevés d'anticorps anti-IFN $\alpha$ comparés aux sujets non répondeurs et placebo (Figure 2C). Chez les patients répondeurs, le risque relatif (RR) de survenue de signes cliniques liés au Sida a été significativement diminué $(R R=0,25 ; p<0,05)$, le nombre de lymphocytes T CD $4^{+}$sanguin $(p<0,03)$ s'est stabilisé et la virémie n'a pas augmenté significativement $(p<0,01)$ [25].

Le vaccin kinoïde étant dirigé contre une cytokine du soi, la réaction immunitaire présente des propriétés inhérentes à son caractère autologue: (1) la réponse anticorps anti-cytokine est transitoire; l'activité neutralisante efficace de ces anticorps disparaît 3 mois après la dernière injection du fait de l'absence d'une mémoire T anti-cytokine. Le maintien de taux d'anticorps efficaces peut cependant être assuré par des injections de rappel, dont la périodicité peut

\begin{tabular}{|c|c|c|}
\hline & $\begin{array}{l}\text { Immunothérapie spécifique } \\
\text { par Ac monoclonaux }\end{array}$ & Vaccination active \\
\hline Thérapeutique & Validée & En cours de validation \\
\hline Principe actif & Anticorps monoclonal & Kinoïde \\
\hline Anticorps anticytokine & Monoclonal & Polyclonal \\
\hline Origine des anticorps (Ac) & $\begin{array}{c}\text { Hybridome ou } \\
\text { synthèse de peptides recombinants }\end{array}$ & Naturelle \\
\hline Taux d'Ac sérique & Élevé & Élevé \\
\hline Réponse cellulaire & Aucune & Aucune \\
\hline Durée de neutralisation de la cytokine & 3 semaines & $2-4$ mois \\
\hline Production d'autoAc anti-Ac & Risque (après injections répétées) & Aucune \\
\hline Fréquence des rappels & Toutes les $1-4$ semaines & Tous les $3-4$ mois \\
\hline Rechute induite par auto-Ac & Possible & Néant \\
\hline Compliance au traitement & Modérée & Bonne \\
\hline
\end{tabular}

Tableau I. Immunothérapie spécifique anticytokine. Comparaison de la vaccination kinoïde à l'immunothérapie spécifique par administration d'Ac monoclonaux. 
être contrôlée par la sérologie. (2) Les anticorps polyclonaux circulants induits par le vaccin ont une forte avidité pour la cytokine

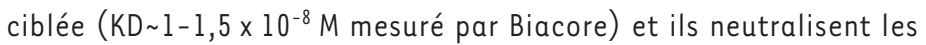
cytokines ectopiques présentes dans les liquides biologiques (sang et lymphe stromale des tissus). Cependant, au niveau des champs cytokiniques (où siègent normalement les réactions cytokiniques), ces anticorps restent sans effet sur la physiologie de ces réactions du fait de la plus forte affinité des récepteurs de cytokine (KD $10^{-11} \mathrm{M}$ ) exprimés à la surface des cellules cibles [26]. (3) Les kinoïdes n'induisent pas de réponse cellulaire T dirigée contre la cytokine du soi ciblée. Ce résultat était anticipé du fait de la sélection clonale centrale thymique négative et de la présence de cellules T régulatrices périphériques contrôlant les antigènes du soi au niveau des organes lymphoïdes. (4) Les kinoïdes KLH-cytokine induisent une réponse anti-KLH, à la fois humorale et cellulaire. Cette réponse collatérale sans effet indésirable immédiat et à long terme [14] serait bénéfique par son action immunomodulatrice [17].

\section{Tolérance au vaccin kinoïde}

Les études expérimentales en clinique réalisées depuis 1992 ont montré une bonne tolérance des vaccins kinoïdes dont l'administration n'a été associée à ce jour à aucune complication locale ou systémique. Ce résultat était prévisible du fait de l'absence de toute toxicité intrinsèque du produit kinoïde et des caractéristiques mentionnées ci-dessus de la réaction immunitaire qu'il induit dont en particulier: (1) l'absence de réaction des lymphocytes T anti-cytokine, qui élimine le risque de pathologie auto-immune, et (2) l'absence de dérèglement des processus physiologiques cytokiniques. Comme cela a été mentionné au paragraphe précédent, l'action des anticorps anti-cytokine, qu'ils soient administrés passivement (anticorps monoclonaux, Acm) ou produits par vaccination kinoïde, s'exerce essentiellement au niveau des cytokines ectopiques circulantes, ce qui réduit le risque de complications que provoquerait un stress exogène ou un stimulus infectieux susceptible d'induire des réactions physiologiques cytokiniques.

Chez l'animal, la vaccination kinoïde réalisée dans différents modèles expérimentaux a toujours été bien tolérée. Des rats immunisés contre l'EGF (epidermal growth factor) ayant développé des auto-anticorps spécifiques de forte affinité n'ont présenté aucune complication et aucune lésion inflammatoire au sein des différents tissus [28]. Chez la souris les immunisations actives anti-IL-1 [29] et anti-IL-9 [18] se sont avérées totalement inoffensives sur une période de suivi supérieur à 12 mois.

Les études précliniques réalisées avec les kinoïdes KLH-TNF- $\alpha$ humain, KLH-IL-4 humain et IFN- $\alpha$ humain inactivé (Antiferon ${ }^{\circledast}$ ) [25] n'ont révélé chez la souris, le chien ou le singe aucune toxicité aiguë ou chronique. Au cours d'essais cliniques réalisés chez les patients atteints de cancers du côlon, de la prostate ou du sein, les vaccins anti-EGF ont été bien tolérés [30]. Dans le cadre de l'essai EURIS multicentrique de phase II/II réalisé en double aveugle sur 242 patients sidéens, l'immunisation par l'IFN- $\alpha$ inactivé (Antiferon ${ }^{\circledR}$ ), qui a produit des titres élevés d'anticorps chez certains sujets, n'a révélé aucun effet collatéral infectieux ou autoimmun inhérent à l'immunisation pendant les 18 mois du suivi clinique [25].

\section{Discussion et conclusion}

La mise en évidence du rôle majeur joué par les dérèglements cytokiniques dans la pathogenèse de maladies chroniques a conduit les scientifiques à développer des stratégies susceptibles d'inhiber spécifiquement les cytokines ectopiques délétères portées par la lymphe des tissus pathologiques. Cette stratégie de ciblage spécifique anti-cytokine s'est montrée efficace et, à I'heure actuelle, les Acm anti-TNF $\alpha$ (Remicade ${ }^{\circledR}$ ou Humira $\left.^{\oplus}\right)[31,32]$ tout comme les récepteurs solubles du TNF- $\alpha$ (Etanercept ${ }^{\circledR}$ ) [33], constituent le socle thérapeutique de maladies auto-immunes telles que la polyarthrite rhumatoïde ou la maladie de Crohn, tandis que les Acm anti-VEGF (Avastin ${ }^{\circledR}$ ) [34] sont associés à la chimiothérapie pour combattre l'angiogenèse et les métastases de cancer. Que ces anticorps spécifiques soient chimériques (Remicade ${ }^{\circledast}$ ), humanisés (Avastin ${ }^{\circledR}$ ) ou humains (Humira ${ }^{\circledR}$ ), ils peuvent induire, après leur administration répétée, des auto-anticorps anti-Acm (anti-idiotypes ou autres), source de récidives qui peuvent en limiter l'usage [35]. Par la faisabilité de leur préparation, leur innocuité et leur efficacité exposées dans cet article, les vaccins kinoïdes pourraient représenter une alternative thérapeutique à l'immunothérapie spécifique par $\mathrm{Acm}$, en particulier en cas de résistance au traitement de ces derniers [36]. Après validation par les essais cliniques en cours de développement ou en préparation par Neovacs, I'utilisation du vaccin kinoïde lors de thérapies anticytokines prolongées pourra présenter des avantages: (1) induction d'anticorps polyclonaux naturels de haute affinité non susceptibles d'induire des autoanticorps contre euxmêmes [35] ; (2) maintien permanent, conformément à la logique des protocoles thérapeutiques métronomiques [37], de titres d'anticorps neutralisants à des niveaux efficaces grâce à des injections de rappel dont la périodicité sera guidée par le suivi sérologique; (3) confort apporté au patient du fait de la faible fréquence des rappels. De plus, le sang de sujets volontaires vaccinés pourra représenter une source unique d'anticorps anti-cytokines neutralisants naturels. Ces derniers, qu'ils soient des immunoglobulines polyclonales purifiées provenant de sérums hyperimmuns ou des anticorps monoclonaux produits à partir de cellules $B$ immortalisées, pourront être utilisés lors d'indication d'immunothérapie passive sans risque de générer des auto-anticorps. Par ses avantages résumés dans le 
Tableau I, la stratégie Kinoïde devrait contribuer à élargir le champ d'application des immunothérapies anti-cytokines validé depuis dix ans grâce aux succès cliniques rencontrés par l'administration des Acm dans le traitement de maladies graves telles la polyarthrite rhumatoïde, la maladie de Crohn ou certains cancers. $\diamond$

\section{GLOSSAIRE}

Ac: Anticorps

Acm : Anticorps monoclonal

CPA : cellule présentatrice d'antigène

CPG : Cytosine-phosphatidyl-guanosine dinucléotides

KLH : keyhole limpet hemocyanin

LPS : Lipopolysaccharide

PDC : plasmacytoïd dendritic cells

SDF-1 : stromal derived factor-1

Sida : Syndrome d'Immunodéficience Acquise

TAA : tumor associated antigen

TGF- $\boldsymbol{\beta}$ : transforming growth factor $\beta$

TNF- $\alpha$ : tumor necrosis factor- $\alpha$

TRAIL: TNF-related apoptosis inducing ligand

TSA : tumor specific antigen

VEGF : vascular endothelial growth factor

VIH-1 : Virus de l'immunodéficience humaine

\section{SUMMARY}

\section{Kinoids: a novel generation}

of specific immune therapy against cytokines

The abnormal cytokine release in the stromal microenvironment of pathologic tissues, contributes to the pathogenesis of viral infections such as AIDS, cancer and auto-immune diseases. Neovacs developed therapeutic vaccines, named Kinoids, which induce anti-cytokine Antibodies. Kinoids are non toxic but immunogenic cytokine derivatives. Kinoid immunizations induce high titre of neutralizing Abs to the corresponding cytokine, is well tolerated and experimentally effective. In transgenic mice expressing huTNF $\alpha$, the TNF $\alpha$ kinoid decreases clinical signs of Rheumatoid Arthritis and in mice challenged with syngenic CT26 tumor cell line huVEGF kinoid inhibits lung metastases. After validation by clinical trials, kinoid vaccines could represent a second generation of specific immune therapy to be used to combat ectopic cytokines. $\diamond$

\section{RÉFÉRENCES}

1. Leclerc C. L'apport des nouvelles technologies en vaccinologie. Med Sci (Paris) 2007 ; $23: 386-90$.

2. Zagury D, Le Buanec $H$, Bizzini B, et al. Active versus passive anti-cytokine antibody therapy against cytokine-associated chronic diseases. Cytokine Growth Factor Rev $2003 ; 14: 123-37$.

3. Belshe RB, Graham BS, Keefer MC, et al. Neutralizing antibodies to HIV-1 in seronegative volunteers immunized with recombinant gp 120 from the MN strain of HIV-1. NIAID Aids vaccine clinical trials network. JAMA $1994 ; 272: 475-80$.

4. Bleumer I, Tiemessen DM, Oosterwijk-Wakka JC, et al. Preliminary analysis of patients with progressive renal cell carcinoma vaccinated with CA9-peptide-pulsed mature dendritic cells. J Immunother $2007 ; 30: 116-22$.

5. Future II Study Group. Quadrivalent vaccine against human papillomavirus to prevent highgrade cervical lesions. $N$ Engl J Med 2007 ; 356 : 1915-27.
6. Orenstein JM, Preble OT, Kind P, Schulof R. The relationship of serum alpha-interferon and ultrastructural markers in HIV-seropositive individuals. Ultrastruct Pathol 1987 ; $11: 673-9$.

7. Badou A, Bennasser $Y$, Moreau M, et al. Tat protein of human immunodeficiency virus type 1 induces interleukin- 10 in human peripheral blood monocytes: implication of protein kinase C-dependent pathway. J Virol $2000 ; 74: 10551-62$.

8. Kekow J, Wachsman W, McCutchan JA, et al. Transforming growth factor beta and noncytopathic mechanisms of immunodeficiency in human immunodeficiency virus infection. Proc Natl Acad Sci USA 1990 ; $87: 8321-5$.

9. Gallo RC. Tat as one key to HIV-induced immune pathogenesis and Tat (correction of Pat) toxoid as an important component of a vaccine. Proc Natl Acad Sci USA $1999 ; 96: 8324-6$.

10. Le Buanec H, D’Anna R, Lachgar A, et al. HPV- 16 ع7 but not $\varepsilon 6$ oncogenic protein triggers both cellular immunosuppression and angiogenic processes. Biomed Pharmacother 1999 ; $53: 424-31$.

11. D'Anna R, Le Buanec H, Alessandri G, et al. Selective activation of cervical microvascular endothelial cells by human papillomavirus $16-\mathrm{e} 7$ oncoprotein. J Natl Cancer Inst 2001 ; 93 : 1843-51.

12. Akagi K, Ikeda $Y$, Miyazaki $M$, et al. Vascular endothelial growth factor-C (VEGF-C) expression in human colorectal cancer tissues. Br J Cancer 2000 ; $83: 887-91$.

13. Feldmann $M$, Steinman L. Design of effective immunotherapy for human autoimmunity. Nature $2005 ; 435: 612-9$

14. Le Buanec H, Delavallee L, Bessis N, et al. TNFalpha kinoid vaccinationinduced neutralizing antibodies to TNFalpha protect mice from autologous TNFalpha-driven chronic and acute inflammation. Proc Natl Acad Sci USA $2006 ; 103$ : 19442-7.

15. Bizzini $B$, Achour A. « Kinoids »: the basis for anticytokine immunization and their use in HIV infection. Cell Mol Biol $1995 ; 41$ : 351-6.

16. Sabbatini PJ, Kudryashov V, Ragupathi G, et al. Immunization of ovarian cancer patients with a synthetic Lewis $(y)$-protein conjugate vaccine: a phase 1 trial. Int J Cancer $2000 ; 87: 79-85$.

17. Lamm DL, Dehaven JI, Riggs DR. Keyhole limpet hemocyanin immunotherapy of bladder cancer: laboratory and clinical studies. Eur Urol $2000 ; 37$ (suppl 3) : 41-4.

18. Richard M, Grencis RK, Humphreys NE, et al. Anti-IL-9 vaccination prevents worm expulsion and blood eosinophilia in Trichuris murisinfected mice. Proc Natl Acad Sci USA 2000 ; 97 : 767-72.

19. Uyttenhove C, Van Snick J. Development of an anti-IL-17A auto-vaccine that prevents experimental auto-immune encephalomyelitis. Eur J Immunol $2006 ; 36: 2868-74$.

20. Chackerian B, Lenz P, Lowy DR, Schiller JT. Determinants of autoantibody induction by conjugated papillomavirus virus-like particles. J Immunol $2002 ; 169: 6120-6$.

21. Galle $P$, Hougs L, Barington T, et al. Knocking out IL-6 by vaccination. Eur J Immunol $2004 ; 34: 291-300$.

22. Spohn G, Guler R, Johansen P, et al. A virus-like particle-based vaccine selectively targeting soluble TNF-alpha protects from arthritis without inducing reactivation of latent tuberculosis. J Immunol $2007 ; 178: 7450-7$.

23. Chensue SW, Terebuh PD, Remick DG, et al. In vivo biologic and immunohistochemical analysis of interleukin-l alpha, beta and tumor necrosis factor during experimental endotoxemia. Kinetics, Kupffer cell expression, and glucocorticoid effects. Am J Pathol 1991; 138 : 395-402.

24. Weiner GJ. The immunobiology and clinical potential of immunostimulatory CpG oligodeoxynucleotides. J Leukoc Biol 2000 ; 68: 455-63.

25. Gringeri A, Musicco M, Hermans P, et al. Active anti-interferon-alpha immunization: a European-Israeli, randomized, double-blind, placebocontrolled clinical trial in 242 HIV-1 infected patients (the EURIS study). J Acquir Immune Defic Syndr Hum Retrovirol 1999 ; 20 : 358-70.

26. Rad FH, Le Buanec H, Paturance $S$, et al. VEGF kinoid vaccine, a therapeutic approach against tumor angiogenesis and metastases. Proc Natl Acad Sci USA 2007 ; 104 : 2837-42.

27. Le Buanec $H$, Paturance $S$, Couillin I, et al. Control of allergic reactions in mice by an active anti-murine IL-4 immunization. Vaccine 2007 ; $25: 7206-16$

28. Raaberg L, Nexo $\varepsilon$, Jorgensen PE, et al. Fetal effects of epidermal growth factor deficiency induced in rats by autoantibodies against epidermal growth factor. Pediatr Res $1995 ; 37$ : 175-81.

29. Svenson M, Hansen MB, Thomsen AR, et al. Cytokine vaccination: neutralising IL-lalpha autoantibodies induced by immunisation with homologous IL-lalpha. J Immunol Methods 2000 ; 236 : 1-8. 
30. Gonzalez G, Crombet T, Catala M, et al. A novel cancer vaccine composed of humanrecombinant epidermal growth factor linked to a carrier protein: report of a pilot clinical trial. Ann Oncol $1998 ; 9: 431-5$.

31. Elliott MJ, Maini RN, Feldmann M, et al. Randomised double-blind comparison of chimeric monoclonal antibody to tumour necrosis factor alpha (cA2) versus placebo in rheumatoid arthritis. Lancet 1994 ; 344 : 1105-10.

32. Den Broeder A, Van de Putte L, Rau R, et al. A single dose, placebo controlled study of the fully human anti-tumor necrosis factor-alpha antibody adalimumab (D2E7) in patients with rheumatoid arthritis. J Rheumatol $2002 ; 29: 2288-98$.

33. Weinblatt ME, Kremer JM, Bankhurst AD, et al. A trial of etanercept, a recombinant tumor necrosis factor receptor:Fc fusion protein, in patients with rheumatoid arthritis receiving methotrexate. N Engl J Med 1999 ; $340: 253-9$.

34. Kabbinavar F, Hurwitz HI, Fehrenbacher L, et al. Phase II, randomized trial comparing bevacizumab plus fluorouracil (FU)/leucovorin (LV) with FU/LV alone in patients with metastatic colorectal cancer. J Clin Oncol 2003; $21: 60-5$.
35. Anderson PJ. Tumor necrosis factor inhibitors: clinical implications of their different immunogenicity profiles. Semin Arthritis Rheum 2005 ; $34: 19-22$.

36. Friedberg JW. Unique toxicities and resistance mechanisms associated with monoclonal antibody therapy. Hematology Am Soc Hematol Educ Program 2005; 329-34.

37. Kerbel RS, Kamen BA. The anti-angiogenic basis of metronomic chemotherapy. Nat Rev Cancer $2004 ; 4: 4$ 23-36.

38. Silbermann B, Launay 0 . Prévention des infections à papillomavirus et du zona: nouveaux vaccins. Med Sci (Paris) $2007 ; 23: 423-7$.

\section{Cycle de 3 conférences-débats :}

\section{L'embryon, le fæetus, l'enfant}

\section{Assistance Médicale à la Procréation (AMP) et lois de bioéthique}

L'embryon, le faxtus et l'enfant sont au cąur des lois de bioéthique, encadrant l'Assistance médicale à la Procréation, dont la révision est prévue en 2009.

L'Institut du droit de la famille et du patrimoine et l'Académie nationale de médecine se sont naturellement associés pour apporter dès à présent au débat leurs expériences respectives et leur réflexion.

Ces questions, outre qu'elles doivent faire l'objet d'échanges transdisciplinaires, ne peuvent plus rester l'apanage des seuls spécialistes. C'est pourquoi les participants aux conférences-débats seront largement associés à l'ensemble des discussions. II s'agit de dépasser les frontières de la médecine et du droit pour permettre justement aux médecins et aux juristes de confronter leurs points de vue à la lumière de la philosophie, des sciences humaines et du débat public.

Ce cycle de conférences est organisé au cours de trois demi-journées, avec des interventions croisées de juristes, de médecins et de représentants des sciences humaines qui font référence sur ces sujets.

L'ensemble des interventions fera l'objet d'une publication par les éditions ESKA.

\section{Maison du barreau}

\section{2-4 rue de Harlay, 75001 Paris}

Lundi 17 mars 2008 - L'embryon in vitro : qui ou quoi ?

LE CHAMP DES POSSIBLES : le pouvoir de procréer avec assistance médicale avec françois THÉPOT,

Frédérique DREIFUSS-NETTER, Jennifer MERCHANT

L'EMBRYON SUJEt/OBJEt DE SOINS Et DE RECHERCHES avec Pierre JOUANNET, Grégoire LOISEAU, Simone BATEMAN

Jeudi 17 avril 2008 - Le færtus dans tous ses états : quel statut ?

LE FGIUS : UN PATIENT ? avec Claude SUREAU, Agnès LOUIS-PECHA, Anne FAGOT-LARGEAULT

LA MATERNITÉ DE SUBSTITUTION avec Paul DEVROEY, Béatrice WEISS-GOUT, Dominique MEHL.

Vendredi 16 mai 2008 - L'enfant issu d'une AMP : quelle filiation?

NOUVEAUX MODES DE PROCRÉATION ET ÉTABLISSEMENT D’UN LIEN DE FILIATION avec Bernard GOLSE, Brigitte FEUILLET-LE MINTIER, Carine CAMBY

PROCRÉATION MÉDICALISÉE : Intervention de l'État et/ou dynamique de marché ? Bilan et perspectives

Synthèse de l'ensemble du cycle par Hervé CHNEIWEISS, Pierre MURAT et Alex MAURON

Clôture des travaux : Madame Valérie PECRESSE, Ministre de la Recherche et de l'Enseignement Supérieur INFORMATIONS ET INSCRIPTION : CFEE - Serge KEBABTCHIEfF - 12 rue du Quatre Septembre - 75002 Paris, Tél : $0142865569 / 87$ Fax : 0142604535 - congres@esha.fr 